\title{
Atividade antibacteriana do extrato hidroalcoólico de Punica granatum Linn. sobre Staphylococcus spp. isolados de leite bovino ${ }^{1}$
}

\author{
Giovanna M.B. Moreira², Leopoldo S. Matsumoto³, Regildo M.G. Silva ${ }^{4}$, Paulo F. \\ Domingues $^{5}$ e Erika C.T. Mello-Peixoto ${ }^{6 *}$
}

\begin{abstract}
Moreira G.M.B., Matsumoto L.S., Silva R.M.G., Domingues P.F. \& Mello-Peixoto E.C.T. 2014. [Antibacterial activity of the hydroalcoholic extract of Punica granatum Linn. on Staphylococcus spp. isolated from bovine milk.] Atividade antibacteriana do extrato hidroalcoólico de Punica granatum Linn. sobre Staphylococcus spp. isolados de leite bovino. Pesquisa Veterinária Brasileira 34(7):626-632. Centro de Ciências Agrárias, Universidade Estadual do Norte do Paraná, Campus Luiz Meneghel, BR-369 Km 54, Vila Maria, Cx. Postal 26, Bandeirantes, PR 86360-000, Brazil. E-mail: emellopeixoto@uenp.edu.br

Bovine mastitis is characterized by inflammation of the mammary gland, usually in response to bacterial infection, affecting qualitatively and quantitatively milk production. This study aimed to determine the antibacterial activity in vitro of the hydroalcoholic extract of the bark of the pomegranate on bacteria isolated from bovine milk. The colonies of Staphylococcus spp. were resuspended in 6 MacFarland scale and adjusted its concentration by UV visible spectrophotometry at a concentration of $10^{6} \mathrm{ml}^{-1}$. The extracts were evaluated in quintuplicate in seven concentrations: from 4 up to $0.0625 \mathrm{mg}^{-\mathrm{mL}^{-1}}$. The sensitivity of microbial isolates was determined using the disk diffusion and the results that showed inhibition halos corresponding to values from $15 \mathrm{~mm}$ were considered susceptible. The results were evaluated by ANOVA, Tukey test $5 \%$ using the SISVAR 5.3-DEX/UFLA. Additionally the extract was assessed as for the antioxidant activity, content of phenols and total flavonoids. The extract was diluted into seven concentrations: $25-1.000 \mu \mathrm{g} \cdot \mathrm{mL}^{-1}$, and evaluated in triplicate. The bacterial growth was inhibited starting from the concentration of 4 mg.mL $\mathrm{L}^{-1}$ and antioxidant activity was observed from $50 \mu \mathrm{g} \cdot \mathrm{mL}^{-1}$, with values corresponding to $4.62 \%$ reaching the plateau of $64.90 \%$ at a concentration of $500 \mu \mathrm{g} \cdot \mathrm{mL}^{-1}$. In evaluating the radical scavenging activity, using the free radical DPPH, the extract demonstrated antioxidant activity $\left(\mathrm{IC}_{50} \%=378.80 \mu \mathrm{g} / \mathrm{mL}\right)$. However it has not been possible to correlate the antioxidant activity with the levels of phenols and flavonoids. Perhaps the presence of other substances alkaloids and tannins present in the extracts may have been responsible for the antioxidant activity found. It was concluded that the hydroalcoholic extract of $\mathrm{Pu}$ nica granatum Linn. has antimicrobial activity against Staphylococcus spp., demonstrating potential benefit for the control of bovine mastitis.
\end{abstract}

INDEX TERMS: Mastitis, antimicrobial, natural extract, medicinal plants, organic production, Pomegranate.

\footnotetext{
${ }^{1}$ Recebido em 28 de fevereiro de 2014.

Aceito para publicação em 12 de maio de 2014.

${ }^{2}$ Mestranda em Agronomia, Universidade Estadual do Norte do Paraná, Campus Luiz Meneghel, BR-369 Km 54, Vila Maria, Cx. Postal 26, Bandeirantes, PR 86360-000, Brasil.

${ }^{3}$ Centro de Ciências Biológicas, Universidade Estadual do Norte do Paraná, Campus Luiz Meneghel, BR-369 Km 54, Vila Maria, Cx. Postal 26, Bandeirantes, PR 86360-000.

${ }^{4}$ Departamento de Ciências Biológicas, Faculdade de Ciências e Letras
}

\footnotetext{
de Assis, Universidade Estadual Paulista Júlio de Mesquita Filho (Unesp), Av. Dom Antônio 2100, Vila Universitária, Assis, SP 19806-900, Brasil.

${ }^{5}$ Departamento de Higiene Veterinária e Saúde Pública, Faculdade de Medicina Veterinária e Zootecnia, Unesp, Distrito de Rubião Junior s/n, Botucatu, SP 18618-970, Brasil.

${ }^{6}$ Centro de Ciências Agrárias, Universidade Estadual do Norte do Paraná, Campus Luiz Meneghel, BR-369 Km 54, Vila Maria, Cx. Postal 26, Bandeirantes, PR 86360-000.*Autor para correspondência: emellopeixoto@ uenp.edu.br
} 
RESUMO.- Mastite bovina é caraterizada por inflamação da glândula mamária, geralmente em resposta à infecção bacteriana, compromete quali-quantitativamente a produção leiteira. Este estudo objetivou verificar a atividade antibacteriana in vitro do extrato hidroalcoólico da casca da romã sobre bactérias isoladas de leite bovino. As colônias de Staphylococcus spp. foram ressuspendidas a escala 6 de MacFarland e ajustada a sua concentração por espectrofotometria UV visível na concentração de $10 \mathrm{~mL}^{-1}$. Os extratos foram avaliados em quintuplicata, em sete concentrações: de $4 \mathrm{mg} \mathrm{mL}^{-1}$ até $0,0625 \mathrm{mg} \cdot \mathrm{mL}^{-1}$. A sensibilidade dos isolados microbianos foi determinada utilizando o teste de difusão em disco e os resultados que apresentaram zonas de inibição correspondentes a valores a partir de $15 \mathrm{~mm}$, foram considerados sensíveis. Os resultados foram avaliados pelo método ANOVA, teste de Tukey 5\%, utilizando o SISVAR 5.3 - DEX/UFLA. Adicionalmente o extrato foi avaliado quanto à atividade antioxidante, teores de fenóis e flavonoides totais. Para tanto o extrato foi diluído em sete concentrações: de 25 a $1000 \mu \mathrm{g} . \mathrm{mL}^{-1}$, e avaliado em triplicata. 0 crescimento bacteriano foi inibido a partir da concentração de $4 \mathrm{mg} \cdot \mathrm{mL}^{-1} \mathrm{e}$ a ação antioxidante foi verificada a partir de $50 \mu \mathrm{g} . \mathrm{mL}^{-1}$, com valores correspondentes a $4.62 \%$, atingindo platô de $64,90 \%$ na concentração de $500 \mu \mathrm{g} \cdot \mathrm{mL}^{-1}$. $\mathrm{Na}$ avaliação da atividade captadora de radicais, empregando o radical livre DPPH, o extrato demonstrou atividade antioxidante $\left(\mathrm{IC}_{50} \%=378,80 \mu \mathrm{g} / \mathrm{mL}\right)$. Porém, não foi possível correlacionar a atividade antioxidante aos teores de fenóis e flavonoides. Talvez a presença de outras substâncias alcaloides e taninos presentes no extrato, possam ter sido as responsáveis pela atividade antioxidante encontrada. Conclui-se que o extrato hidroalcoólico de Punica granatum Linn. apresenta atividade antimicrobiana contra Staphylococcus spp., demonstrando potencial benefício para o controle da mastite bovina.

TERMOS DE INDEXAÇÃO: Mastite, antimicrobiano, extrato natural, plantas medicinais, produção orgânica, romã.

\section{INTRODUÇÃO}

Mastite bovina é uma doença multifatorial, caraterizada por inflamação da glândula mamária, frequentemente em resposta à infecção bacteriana, podendo ser causada por outros micro-organismos como algas e fungos (Tozzetti et al. 2008). Em virtude desta infecção, o risco de agentes patogênicos é eminente, uma vez que o leite produzido por animais portadores de mastite apresenta potencial de contaminação por abrigar elevado número de micro-organismos (Ruegg 2003). Além disso, ocasiona prejuízos ao produtor e laticínios, gerando gastos excessivos com medicamentos, mão-de-obra qualificada e serviços veterinários, além da redução da qualidade e quantidade do leite produzido (Feßler et al. 2012, Langoni 2013).

A forma subclínica é principalmente transmitida durante a ordenha por patógenos adaptados à glândula mamária, como Staphylococcus aureus, Streptococcus agalactiae, Streptococcus dysgalactiae e Streptococcus uberis (Oliveira et al. 2011, Mello-Peixoto et al. 2012, Saeki et al. 2012). Esses micro-organismos são disseminados principalmente pelas mãos dos ordenhadores e pelas teteiras contaminadas (Colombo et al. 2012, Raza et al. 2013). S. aureus é frequentemente incriminado como principal agente etiológico na mastite bovina (Marques et al. 2013, Ondiek et al. 2013).

Manejo adequado, higienização na ordenha e antissepsia dos tetos, são medidas que contribuem profilaticamente para amenizar o problema (Haftu et al. 2012, Langoni 2013). A imersão de tetos em antissépticos pode reduzir novas infecções em 50 a 90\% (Pedrini \& Margatho 2003).

0 tratamento da forma clínica é à base de antimicrobianos químicos, entretanto, seu uso na lactação, no controle da mastite subclínica é pouco frequente entre os produtores, principalmente pela baixa eficácia e necessidade de descarte do leite pela presença de resíduos de antibióticos (Brasil 2002). Dessa forma, determinou-se a busca por soluções que trate do problema sem gerar descarte do produto. A tendência de exigência de alto padrão de qualidade da produção do leite e seus derivados, determina cada vez mais a busca por alternativas naturais de tratamento (Trevisan et al. 2009). A utilização de extratos de ervas medicinais tem sido encorajada nas diversas atividades da agropecuária (Souza et al. 2013).

Nos últimos anos, o aumento pela procura de produtos orgânicos vem se intensificando. A utilização de plantas medicinais vem cada vez mais sendo estimulada pela Organização Mundial da Saúde (OMS), por ser acessível economicamente a grande parte da população. Em torno de $80 \%$ da população dos países em desenvolvimento utiliza deste tipo de medicamento (Galdino et al. 2012, Souza et al. 2013).

Punica granatum Linn., conhecida como romanzeira, espécie da família Punicaceae, é cultivada mundialmente (Rummun et al. 2013). Foram demonstradas propriedades antimicrobianas do extrato de Punica granatum Lin. (Jain \& Nafis 2011, Almeida et al. 2012, Moorthy et al. 2013, Rajan et al. 2013), inclusive sobre isolados de S. aureus (Menezes et al. 2008, Oliveira et al. 2010, Hayouni et al. 2011, Moorthy et al. 2013).

Os principais constituintes da romã são alcaloides (peletierina, isopeletierina, metilpeletierina), taninos, compostos fenólicos (antocianinas, quercetina, ácidos fenólicos) e flavonoides (Lansky \& Newman 2007), substâncias frequentemente relacionadas como aquelas responsáveis pelas atividades terapêuticas. Propriedades antibacterianas e anti-inflamatórias apresentadas pelos extratos de romã são particularmente importantes para o tratamento da mastite bovina (Lee et al. 2010, Ismail et al. 2012).

Dessa forma, o presente trabalho objetivou avaliar os efeitos in vitro do extrato hidroalcoólico da casca do fruto da romã sobre Staphylococcus spp. isolados de leite bovino.

\section{MATERIAL E MÉTODOS}

Os frutos de Punica granatum Linn. foram colhidos ao final da manhã no município de Bandeirantes - Paraná. Uma amostra do vegetal foi herborizada e identificada no Instituto Florestal de Assis/SP, e uma exsicata foi depositada no Herbário do Instituto sob o número SPSF 40136.

Os frutos foram higienizados com solução aquosa de hipoclorito de sódio a 0,5\%, secos com papel toalha e descascados. 
As cascas foram acondicionadas em papel $\mathrm{Craft}^{\circledR}$ e encaminhadas para secagem em estufa de ventilação forçada de ar a $40^{\circ} \mathrm{C}$. Posteriormente, foi realizada maceração manual e pesagem para obtenção do extrato hidroalcoólico das cascas de romã a $10 \%$. Foram utilizados $30 \mathrm{~g}$ do material vegetal em $190 \mathrm{~mL}$ de álcool P.A. e $80 \mathrm{~mL}$ de água destilada. Após procedeu-se agitação mecânica constante por 24 horas, com posterior filtração à vacuo. A fim de promover melhor aproveitamento do material vegetal, este procedimento foi realizado por três vezes consecutivas, adicionando-se a cada uma delas a mesma solução hidroalcóolica no mesmo volume supracitado. Posteriormente, o extrato foi concentado por rotaevaporizador, em temperatura de $60^{\circ} \mathrm{C}$ e secagem em estufa a $37^{\circ} \mathrm{C}$ por 24 horas. Após, foi procedido congelamento e liofilização.

Para seleção das amostras de leite portadoras de bactérias do gênero Staphylococcus, foram analisadas 64 amostras de leite, proveniente de animais em lactação, apresentando reação positiva para o exame Califórnia Mastite Teste. A partir destas amostras as bactérias foram isoladas utilizando meio de cultura seletivo Ágar Baird Parker ${ }^{\circledR}$ enriquecido com emulsão de gema de ovo com telurito. Após incubação a $37^{\circ} \mathrm{C}$ por 48 horas, observou-se morfologia, coloração e aspectos macroscópicos das colônias bacterianas, como coloração cinza-negra, brilhante, convexa, com zona de precipitação circundada por halo claro de $2-5 \mathrm{~mm}$. Em meio Ágar Sangue, foram observados aspectos quanto à morfologia, tamanho, pigmentação e presença de hemólise.

As colônias isoladas nos meios de culturas foram observadas em lâminas e coradas por técnica de Gram, as colônias de cocos Gram positivas foram identificadas conforme a produção de catalase. As colônias que apresentaram positividade para o teste da catalase, também foram submetidas ao teste de produção de coagulase (Harmon \& Langlois 1989). As colônias identificadas como coagulase negativa foram submetidas a testes de resistência à novobiocina e teste de urease (Schleifer \& Kloos 1975, Baker et al. 1986, Santos et al. 2010). As colônias identificadas foram contadas e determinadas em Log da unidade formadora de colônias por mililitros de leite ( $\operatorname{LogUFC} \mathrm{mL}^{-1}$ ).

Para avaliação da atividade antimicrobiana foram utilizados os seguintes micro-organismos: S. aureus ATCC 25923, Staphylococcus saprophyticus ATCC 15305 e os isolados S. aureus (SA), Staphylococcus coagulase negativa (SCN) e S. saprophyticus (SS). Foi utilizado a técnica de difusão em discos, utilizando-se placas de Ágar Mueller-Hinton (CLSI/NCCLS 2006). As colônias de Staphylococcus spp. isoladas foram ressuspendidas em solução salina $0,85 \%$ estéril na concentração de 6 na escala de MacFarland e ajustada a concentração de $10^{6} \mathrm{UFC} \mathrm{mL}^{-1}$ por espectrofotometria de luz, em comprimento de onda de $600 \mathrm{~nm}$ com absorbância de 0,40 a 0,49 . Após a secagem, os discos foram fixados sobre o meio Ágar Mueller-Hinton previamente semeado com auxílio de suabe estéril pelos inóculos bacterianos (Pinto et al. 2001). Como controle negativo utilizou-se papel filtro embebido com água destilada estéril. As placas foram incubadas a $37^{\circ} \mathrm{C}$ por 24 a 48 horas e as análises foram realizadas em quintuplicata.

0 extrato liofilizado foi ressuspendido em água destilada estéril na concentração inicial de $4 \mathrm{mg} \mathrm{mL}^{-1}$, e a partir desta solução foram realizadas as seguintes diluições: $2 \mathrm{mg} \mathrm{mL}^{-1}, 1 \mathrm{mg} \mathrm{mL}^{-1}$, $0,5 \mathrm{mg} \mathrm{mL}^{-1}, 0,25 \mathrm{mg} \mathrm{mL}^{-1}, 0,125 \mathrm{mg} \mathrm{mL}^{-1}, 0,0625 \mathrm{mg} \mathrm{mL}^{-1}$. Subsequentemente uma alíquota $40 \mu \mathrm{L}$ de cada extrato foi impregnada em discos de papel filtro (Whatman $\mathrm{n}^{\circ}$ 1), de $7 \mathrm{~mm}$ de diâmetro (Pereira et al. 2009).

A sensibilidade dos isolados microbianos foi determinada utilizando o teste de difusão em disco e os resultados que apresentaram zonas de inibição correspondentes a valores a partir de $15 \mathrm{~mm}$, foram considerados sensíveis. Esses halos foram medidos em milímetros ( $\mathrm{mm}$ ) em relação ao seu diâmetro, com auxílio de régua milimetrada. Os resultados foram submetidos à análise de variância (ANOVA) e as médias comparadas pelo teste de Tukey a 5\% de probabilidade (Barbosa \& Vieira 2011, Barros et al. 2011).

Como padrão de comparação da eficiência dos extratos foram realizados testes de sensibilidade aos fármacos químicos rotineiramente utilizados na clínica veterinária, tais como: ampicilina $(10 \mu \mathrm{g})$, sulfametoxazol e trimetoprim $(25 \mu \mathrm{g})$, penicilina $(10 \mu \mathrm{g})$, oxacilina $(1 \mu \mathrm{g})$ e tetraciclina $(30 \mu \mathrm{g})$ (Pereira et al. 2009).

Em relação aos aspectos fitoquímicos, o extrato hidroalcoólico das cascas do fruto de Punica granatum Linn., foi avaliado quanto à atividade antioxidante, teores de fenóis e flavonoides totais. Para realização dessas determinações, o extrato foi diluído nas concentrações de $25,50,75,100,250,500$ e $1000 \mu$ g para cada $1 \mathrm{~mL}$, e avaliados em triplicata.

A atividade antioxidante (AA\%) do extrato foi determinada pela capacidade doadora de $\mathrm{H}^{+}$para o radical estável DPPH (2,2-difenil-1-picrilhidrazil), de acordo com a metodologia in vitro proposta por Blois (1958). Este método é baseado na redução do radical livre estável DPPH de coloração violeta à DPPH, de coloração amarelada. 0 extrato reage com o radical DPPH em ambiente de pouca luminosidade, em seguida é submetido ao espectrofotômetro ultravioleta visível (UV-Vis) a um comprimento de onda de $517 \mathrm{~nm}$. (Brand-Williamset al. 1995). 0 cálculo da atividade antioxidante foi realizado de acordo com a fórmula: Atividade antioxidante $(\%)=[$ (Acontrole - Aamostra $) /$ Acontrole] x 100 onde Aamostra é a absorbância das amostras após 30 minutos e Acontrole é a absorbância do DPPH; ambos a $517 \mathrm{~nm}$. 0 teste é sensível para detectar baixas concentrações dos princípios ativos (Manian et al. 2008), e o resultado pode ser visualizado pelo grau de descoloração do reagente após os 30 minutos necessários para a reação atingir o estado de platô. A determinação da $\mathrm{IC}_{50}$, ou seja, concentração da amostra ou padrão que causa 50\% de inibição da concentração inicial de DPPH, foi obtida por regressão linear dos pontos plotados graficamente. Para a plotagem dos pontos, foram utilizados os valores das médias obtidas de triplicatas realizadas para o teste DPPH (Di Mambro \& Fonseca 2005).

Para determinação de fenóis totais, o método utilizado foi o de Folin-Ciocalteu (molibdato, tungstato e ácido fosfórico), utilizando ácido gálico como padrão de comparação. A cada $0,5 \mathrm{~mL}$ de extrato, foram adicionados $5 \mathrm{~mL}$ de água destilada e $0,25 \mathrm{~mL}$ do reagente de Folin-Ciocalteu (molibdato, tungstato e ácido fosfórico). Após 3 minutos, foi adicionado $1 \mathrm{~mL}$ de solução de $\mathrm{Na}_{2} \mathrm{CO}_{3}$ saturada a $10 \%$, e a mistura armazenada por 1 hora. A absorbância foi medida a $725 \mathrm{~nm}$ usando um espectrofotômetro UV-Vis. Os resultados foram expressos em $\mathrm{mg}$ de ácido gálico por $\mathrm{g}$ de extrato. 0 ácido gálico é percursor de diversos tipos de compostos fenólicos, possui estrutura simples, e por este motivo é considerado substância de escolha como padrão.

A dosagem dos flavonoides totais do extrato foi determinada por espectrofotômetro UV-Vis. As amostras foram preparadas segundo a metodologia de Zhishen et al. (1999), baseado na complexação dos flavonoides com $\mathrm{AlCl}_{3}$, ocorrendo deslocamento das bandas de absorção para maiores comprimentos de onda. Uma alíquota de $250 \mu \mathrm{L}$ dos extratos foi adicionada a 1,25mL de água destilada e $75 \mu \mathrm{L}$ de solução de $\mathrm{NaNO}_{2}$ a $5 \%$. Após 6 minutos, $150 \mu \mathrm{L}$ de solução de $\mathrm{AlCl}_{3} / \mathrm{H}_{2} \mathrm{O}$ a $10 \%$ foi adicionada. Após $5 \mathrm{mi}-$ nutos, 0 ,5mL de solução de $\mathrm{NaOH} 1 \mathrm{M}$ foi adicionada, e então o volume total completado com $2,5 \mathrm{~mL}$ de água destilada. As amostras foram agitadas em vórtex e a absorbância mensurada a $510 \mathrm{~nm}$. Os resultados foram expressos em mg de rutina por g de extrato. A rutina, assim como a quercetina, apresenta estrutura básica de flavonoides, podendo ser empregada como indicador indireto para este grupo de compostos. 


\section{RESULTADOS}

Para as amostras de leite utilizadas pelo presente estudo, foram identificados 13 isolados de Staphylococcus aureus, 2 isolados de $S$. coagulase negativa e 3 isolados de S. saprophyticus (1, 2 e 3).

Em relação aos resultados referentes à técnica de difusão em discos, o extrato hidroalcoólico de romã a $10 \%$ apresentou atividade antibacteriana. Todos os isolados e as bactérias de referência apresentaram halo de inibição superior que $15 \mathrm{~mm}$; a partir da concentração de $0,125 \mathrm{mg}$ $\mathrm{mL}^{-1}$ (Quadro 1); ao contrário o grupo controle negativo que não apresentou ação inibitória, tão pouco interferência no crescimento microbiano.

Em relação à sensibilidade aos antibióticos químicos testados, todos Staphylococcus spp. apresentaram sensibilidade, com exceção do S. aureus ATCC 25923, que apresentou resistência à tetraciclina. Da mesma forma, todos os isolados de $S$. saprophyticus foram resistentes à tetraciclina (Quadro 2).

O extrato hidroalcoólico de Punica granatum Linn. apresentou atividade antioxidante a partir da concentração de $50 \mu \mathrm{g} . \mathrm{mL}^{-1}$, com valores correspondentes a $4,62 \%$, atingindo $64,90 \%$ na concentração de $500 \mu \mathrm{g} \cdot \mathrm{mL}^{-1}$. 0 extrato apresentou alta atividade antioxidante com $\mathrm{IC}_{50} \%$ correspondendo a $378,80 \mu \mathrm{g} / \mathrm{mL}$. As outras concentrações utilizadas

Quadro 1. Halos de inibição (mm) de crescimento dos Staphylococcus spp. referência (Staphylococcus aureus ATCC 25923 e Staphylococcus saprophyticus ATCC 15305) e isolados (Staphylococcus aureus, Staphylococcus coagulase negativa e Staphylococcus saprophyticus isolados 1, 2 e 3), submetidos às diferentes concentrações de extrato aquoso de Punica granatum Linn. a 10\%

Extrato S. aureus S. saprophyticus S. aureus S. coagulase S. saprophyticus $\mathrm{mg} \mathrm{mL}^{-1}$ ATCC 25923 ATCC $15305 \quad$ negativa $1 \frac{3}{n}$

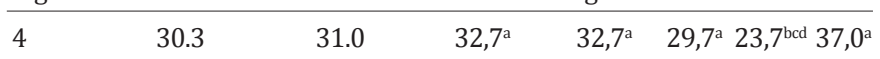

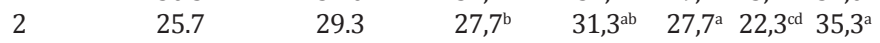

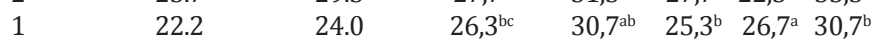

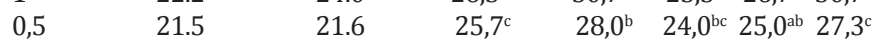

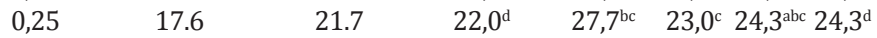

$\begin{array}{llllllll}0,125 & 16.0 & 20.8 & 21,3^{\text {d }} & 23,3^{\mathrm{c}} & 18,0^{\mathrm{d}} & 21,7^{\mathrm{d}} & 24,3^{\mathrm{d}}\end{array}$

$\begin{array}{llllllll}0,0625 & 15.3 & 17.1 & 16,3^{\mathrm{e}} & 9,3^{\mathrm{d}} & 10,0^{\mathrm{e}} & 17,3^{\mathrm{e}} & 16,0^{\mathrm{e}}\end{array}$

Médias seguidas de mesma letra minúscula na coluna não diferem significativamente entre si pelo teste de Tukey a $5 \%$ de probabilidade

Quadro 2. Halos de inibição (mm) de crescimento dos Staphylococcus spp. referência (Staphylococcus aureus ATCC 25923 e Staphylococcus saprophyticus ATCC 15305) e isolados (Staphylococcus aureus, Staphylococcus coagulase negativa e Staphylococcus saprophyticus isolados 1, 2 e 3), submetidos aos fármacos químicos

\begin{tabular}{lcccccccc}
\hline Antibió- & $\begin{array}{c}\text { S.aureus } \\
\text { ticos }^{\text {a }}\end{array}$ & $\begin{array}{c}\text { S. saprophyticus } \\
\text { ATCC } 25923\end{array}$ & S. aureus & S. coagulase & \multicolumn{3}{c}{ S. saprophyticus } \\
\cline { 6 - 8 } & ATCC 15305 & & negativa & 1 & 2 & 3 \\
\hline AMP 10 & 38.0 & 36.0 & 32 & 30 & 36 & 32 & 30 \\
SUT 25 & 32.0 & 36.0 & 30 & 32 & 31 & 28 & 31 \\
OXA 1 & 31.0 & 19.0 & 28 & 18 & 18 & 19 & 21 \\
PEN 10 & 43.0 & 35.0 & 40 & 31 & 30 & 35 & 29 \\
TET 30 & $12.0^{\mathrm{b}}$ & 33.0 & 25 & 26 & $11^{\mathrm{b}}$ & $11^{\mathrm{b}}$ & $11^{\mathrm{b}}$
\end{tabular}

a AMP $10=$ Ampicilina $10 \mu \mathrm{g}$, SUT $=$ Sulfametoxazol + Trimetropim $23,75 \mu \mathrm{g} / 1,25 \mu \mathrm{g}), \mathrm{OXA}=0$ xacilina $1 \mu \mathrm{g}$, PEN $10=$ Penicilina $10 \mathrm{un}$.), TET

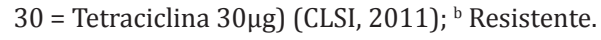

Quadro 3. Valores médios referentes à atividade antioxidante (AA\%) para as diferentes concentrações em $\mu \mathrm{g} . \mathrm{mL}-1$ do Extrato Hidroalcoólico de Punica granatum Linn.

\begin{tabular}{lc}
\hline Concentração $\mu \mathrm{g} \cdot \mathrm{mL}-1$ & $(\mathrm{AA} \%)$ \\
\hline 25 & 0 \\
50 & 4,62 \\
75 & 4,42 \\
100 & 32,99 \\
250 & 35,59 \\
500 & 64,90 \\
1000 & 78,35
\end{tabular}

nesta análise também apresentaram atividade (Quadro 3), entretanto, a atividade antioxidante não foi correlacionada aos teores de fenóis totais e flavonoides.

\section{DISCUSSÃO}

Em referência aos micro-organismos causadores de mastite bovina, Staphylococcus aureus destaca-se como principal agente etiológico da forma subclínica da doença (Ribeiro et al. 2009, Mello-Peixoto et al. 2012, Saeki et al. 2012, Marques et al. 2013). Esta espécie é considerada a mais virulenta do gênero Staphylococcus, devido à formação de toxinas, enzimas mediadoras de invasão tecidual, e sobrevivência no sítio da infecção devido à formação de biofilmes (Raza et al. 2013). Dessa forma, S. aureus pode causar infecções de longa duração com tendência para cronicidade, apresentando baixas taxas de cura (Sabour et al. 2004). Estes fatores, quando analisados conjuntamente com a necessidade de descarte do leite, pela presença de resíduos de antimicrobianos químicos, dificultam a intervenção terapêutica durante a lactação.

Entretanto, o não tratamento da mastite tem sido reavaliado, pois o dano tecidual à glândula mamária é mínimo e reversível durante os estágios iniciais de infecção por $S$. aureus e, se efetivamente tratado neste período, o quarto mamário retornará à produção próxima da normal nas lactações seguintes (Nickerson 1993). Assim, a avaliação de terapêuticas naturais se justifica, principalmente pela possibilidade de se demonstrar potencial benefício para o controle da mastite bovina. Os resultados deste estudo confirmam a eficácia do extrato de romã como potencial agente antibacteriano sobre $S$. aureus, corroborando aos resultados registrados por Catão et al. (2006), Pereira et al. (2006), Silva et al. (2008), Saeki et al. (2012), Moorthy et al. (2013) e Silva et al. (2013).

Catão et al. (2006) compararam a eficiência de antimicrobianos usados rotineiramente na clínica médica aos resultados apresentados pelo extrato etanólico de romã a $10 \%$. Avaliaram 17 cepas de S. aureus de origem humana ambulatorial e registraram que o extrato de romã foi capaz de inibir $100 \%$ das cepas analisadas, enquanto que $64,7 \%$ apresentaram resistência à penicilina e à ampicilina.

Pereira et al. (2006) avaliaram extrato da casca da romã em biofilme dental, verificando a sensibilidade de Streptococcus mitis, Streptococcus mutans, Streptococcus sanguis, Streptococcus sobrinus e Lactobacillus casei. Considerando que um dos componentes mais utilizados para antissepsia oral corresponde à clorexidina, esses pesquisadores re- 
alizaram os mesmos procedimentos com a clorexidina, e registraram que o extrato de romã apresentou melhores resultados. Silva et al. (2008) também verificaram atividade antimicrobiana do extrato da casca do fruto da romã. Avaliaram 38 cepas de S. aureus, sendo que 22 delas eram resistentes à penicilina. Semelhantemente aos resultados encontrados pelo presente estudo, esses autores observaram halos de inibição variando de 10 a $36 \mathrm{~mm}$ de diâmetro.

Em relação aos resultados apresentados pelo teste de difusão em disco, o valor de $15 \mathrm{~mm}$ foi selecionado considerando-se que o limite para o controle laboratorial de $S$. aureus (ATCC 25923), para diferentes antibióticos químicos testados, não ultrapassou $15 \mathrm{~mm}$ diâmetro. Pereira et al. (2006); Saeki et al. (2012) e Silva et al. (2013) também verificaram halos de inibição maiores que $15 \mathrm{~mm}$. Moorthy et al. (2013) utilizaram teste de difusão em discos para avaliar extrato etanólico da casca de Punica granatum Linn. sobre 21 micro-organismos. Avaliaram 250, 500, 750, 1000 e $1250 \mu \mathrm{g} /$ disco, e verificaram inibição do crescimento de mais de $95 \%$ dos micro-organismos testados. Para $S$. aureus, Staphylococcus epidermidis e $S$. mutans, os halos de inibição apresentaram valores de $19.2 \pm 1.15,24.6 \pm 0.87$ e $16.2 \pm 1.31 \mathrm{~mm}$, respectivamente.

Os efeitos do extrato de romã foram testados especificamente contra micro-organismos coletados a partir de animais portadores de mastite bovina (Gopinath et al. 2011, Saeki et al. 2012, Silva et al. 2013). Gopinath et al. (2011) avaliaram a atividade antimicrobiana dos extratos de Cymbopogon citrates, Punica granatum L, Pennisetum setaceum e Nerium oleander. Esses autores concluíram que os extratos de Citratos cymbopogon e Punica granatum L apresentaram atividade antibacteriana, sendo que a maior potência foi observada para o extrato de Punica granatum L. verificarando halos de inibição de 36, 25, 27 e $32 \mathrm{~mm}$ para S. aureus, S. uberis, Escherichia coli e Staphylococcus coagulase negativa, respectivamente.

Em relação à sensibilidade frente aos antibióticos químicos, este estudo verificou resultados semelhantes aos encontrados por Fontana et al. (2010), Saraiva (2012) e Moorthy et al. (2013) que obtiveram 100\% de inibição das cepas frente aos antibióticos testados.

A atividade antimicrobiana é particularmente importante para o tratamento da mastite bovina, uma vez que esta é frequentemente causada em resposta à infecção bacteriana (Oliveira et al. 2011, Mello-Peixoto et al. 2012, Saeki et al. 2012). Da mesma forma, a avaliação da atividade antioxidante é relevante, principalmente para o controle da forma subclínica da doença, pelo fato de que muitas substâncias naturais obtidas de plantas medicinais têm sido identificadas como captadoras de espécies reativas de oxigênio, protegendo o organismo dos efeitos destes, bem como retardando o aparecimento de alterações celulares degenerativas frequentemente presentes em doenças crônicas (Bianchi \& Antunes 1999). Considerando-se que a mastite subclínica rotineiramente é tratada apenas no período de secagem, infectando os animais durante toda lactação, esta forma de apresentação é caracterizada como condição crônica (Sabour et al. 2004), capaz de gerar alterações celulares degenerativas. Assim, se fez relevante para o presente estudo, avaliar a ação antioxidante do extrato de romã. Da mesma forma, a avaliação dos teores de fenóis e flavonoides totais é relevante ao se considerar a capacidade de inibição bacteriana apresentada por estes compostos (Duman et al. 2009, Al-Zahrani 2012). Fenóis e flavonoides auxiliam na ruptura da membrana plasmática, na desnaturação das proteínas e desativação das enzimas auxiliando na ação antimicrobiana de Punica granatum Linn. (Arif et al. 2011).

Assim como o presente estudo Silva et al. (2013) avaliaram extrato de Punica granatum Linn. sobre cepas isoladas de $S$. aureus provenientes de animais portadores de mastite bovina subclínica. Porém, esses autores avaliaram extrato aquoso, seco e in natura, das folhas e cascas dos frutos. Dentre esses extratos, o extrato seco das cascas de romã apresentou os melhores resultados, e bastante semelhantes aos encontrados neste estudo. Porém, apesar desta alta atividade antioxidante encontrada, a mesma não pôde ser correlacionada as teores de fenóis e flavonoides totais.

Manasathien et al. (2012) avaliaram extrato etanólico e aquoso da casca do fruto. Para fenóis totais determinaram os valores de 449,60 GAE.mg-1 e 380,54 GAE.mg-1 respectivamente. Para os teores flavonoides, esses autores encontraram valores de 38,44 GAE.mg-1 e 26,04 GAE.mg-1 respectivamente. Morais et al. (2013) avaliaram o extrato etanólico de Punica granatum Linn. e relacionaram a atividade antioxidante encontrada ao teor de fenóis totais que correspondeu a 135,89 GAE.mg-1. Rummun et al. (2013) encontraram no extrato metanólico da casca do fruto de romã, valores de fenóis totais de 190,27 GAE.g-1 (ácido gálico equivalente) e de flavonoides de 180,10 QE.g-1 (quercetina equivalente). Esses resultados foram obtidos a partir das mesmas concentrações avaliadas pelo presente estudo, porém a metodologia utilizada para a extração dos princípios ativos foi diferenciada. Talvez este fato seja responsável pela diferença de valores encontrados nas pesquisas supracitadas. Punica granatum Linn. apresenta composição química extremamente complexa, com atividade terapêutica específica para cada composto. Talvez outras substâncias alcaloides presente no extrato estudado, possam ter sido as responsáveis pela atividade antioxidante verificada (Moorthy et al. 2013, Rummun et al. 2013).

Machado et al. (2003) e Moorthy et al. (2013) referiram a punicalagina, um tanino elágico derivado do fruto, como um dos principais constituintes antimicrobianos da fruta. Noda et al. (2002) avaliaram extrato acetônico de romã, apontando que para atividade antioxidante, há a contribuição de três antocianinas (delfinidina, cianidina e pelargonidine).

De qualquer forma, e a partir da inibição bacteriana verificada, os resultados apresentados pelo presente estudo permitem destacar o potencial terapêutico do extrato de romã para o controle da mastite bovina. Assim, a utilização de produtos naturais, principalmente a confecção de fitoterápicos a partir da romã, pode representar alternativa importante para o controle da sanidade animal, principalmente para sistemas de produção orgânica ou biológico dinâmica, onde é proibitiva a utilização de medicamentos químicos. Importante destacar que a avaliação do perfil de sensibilidade e de resistência antimicrobiana destes organismos, é primordial à implantação de um sistema de controle eficiente (Chagas 
et al. 2012, Langoni 2013). Adicionalmente, Werkman et al. (2008) consideraram que o uso da romãzeira, pode ser realizado de forma simples sem comprometimento das propriedades biológicas, e que estaria em concordância com as recomendações da OMS quanto ao uso de fontes naturais de baixo custo para o tratamento das afecções.

\section{CONCLUSÃO}

O extrato de Punica granatum Linn. apresentou ação inibitória sobre bactérias do gênero Staphylococcus, principalmente sobre $S$. aureus, demonstrando potencial benefício para o controle da mastite bovina.

Agradecimentos.- À Coordenação de Aperfeiçoamento de Pessoal de Nível Superior (CAPES), pela concessão de bolsa de mestrado, ao Conselho Nacional de Desenvolvimento Científico e Tecnológico (CNPq), aos Ministérios MCTI, MDA, MAPA, MPA e MEC pelo apoio financeiro essencial para a realização deste trabalho.

\section{REFERÊNCIAS}

Almeida L.F.D., Cavalcanti Y.W., Lira Júnior R., Oliveira L.E. \& Casto R.D. 2012. Efeito antifúngico de tinturas de propólis e romã sobre espécies de Candida. Revta Cub. Estomat. 49:99-106.

Al-Zahrani S.H.M. 2012. Antibacterial activities of gallic acid and gallic acid methyl ester on methicillin-resistant Staphylococcus aureus. J. Am. Sci. 8:2.

Arif T., Mandal T.K. \& Dabur R. 2011. Natural products: Anti-fungal agents derived from plants.Opportunity, Challenge and Scope of Natural Prod. Med. Chemistry 81:283-311.

Baker J.S., Hackett M.F. \& Simard D.J. 1986. Variations in bacitracin susceptibility observed in Staphylococcus and Micrococcus species. J. Clin. Microbiol. 23:963-964.

Barbosa M.S. \& Vieira G.H.C. 2011. Diagnóstico e prevenção de mastite em vacas leiteiras no município de Cassilândia/MS. Anais 1ํㅗemex, Dourados, MS. p.3. (Resumo)

Barros J.D., Souza Filho S., Castro V., Torres V.M., Higino J.S. \& Melo A.F.M. 2011. Estudo toxicológico pré-clínico agudo e determinação da CL50 do extrato bruto seco da Vitex agnus castus Linn. Revta Eletr. Farmácia 7:10.

Bianchi M.L.P. \& Antunes L.M.G. 1999. Radicais livres e os principais antioxidantes da dieta. Revta Nutr. 12:123-130.

Blois M.S. 1958. Antioxidant determinations by the use of a stable free radical. Nature 181:1199-1200.

Brand-Williams W., Cuvelier M. \& Berset C. 1995. Use of a free radical method to evaluate antioxidant activity. LWT Technol. 28:25-30.

Brasil 2002. Regulamentos técnicos de produção, identidade, qualidade coleta e transporte de leite. Instrução Normativa no 051 de 18 de setembro de 2002, Ministério de Agricultura, Pecuária e Abastecimento, Brasília.

Catão R.M.R., Antunes R.M.P., Arruda T.A., Pereira M.S.V., Higino J.S., Alves J.A., Passos M.G.V.M. \& Santos V.L. 2006. Atividade antimicrobiana. Revta Bras. Anal. Clin. 38:111-114.

CLSI 2006. Performance Standards for Antimicrobial Ausceptibility Testing. Clinical and Laboratory Standards Institute (NCCLS). Fifteenth Informational Supplement. CLSI/NCCLS document M100-S15.

CLSI 2011. Suggested Grouping of US-FDA Approved Antimicrobial Agents That Should Be Considered for Routine Testing and Reporting on Nonfastidious Organisms by Clinical Laboratories, publication. CLSI/NCCLS document M100-S21.

Chagas L.G.S., Melo P.C., Barbosa N.G., Guimarães E.C. \& Brito D.V.D. 2012. Ocorrência de mastite bovina causada por Staphylococcus sp., Streptococcus sp. e Candida sp. em uma propriedade rural no município de Indianópolis-Minas Gerais, Brasil. Biosci. J. 28:1007-1014.

Colombo R.B., Silva A.V., Martins L.A., Mello P.L., Agostinis R.O. \& Barzon E.M. 2012. Prevalência da mastite subclínica e associação dos agentes etiológicos com a contagem de células somáticas de vacas leiteiras da região sudoeste do Paraná. Vet. Zootec. 19:513-521.

Di Mambro V.M. \& Fonseca M.J.V. 2005. Assays of physical stability and antioxidant activity of a topical formulation added with different plant extracts. J. Pharmaceut. Biomed. Analysis 37:287-295.

Duman A.D., Ozgen M., Dayisoylu K.S., Erbil N. \& Durgac C. 2009. Antimicrobial activity of six pomegranate (Punica granatum L.) varieties and their relation to some of their pomological and phytonutrient characteristics. Molecules 14:1808-1817.

Feßler A.T., Kaspar H., Lindeman C.J., Stegemann M.R., Peters T., Mankertz J., Watts J.L. \& Schwarz S. 2012. A proposal of interpretive criteria for cefoperazone applicable to bovine mastitis pathogens. Vet. Microbiol. 157:226-231.

Fontana V.L.D.S., Giannini M.J.S.M., Leite C.Q.F., Miranda E.T., Almeida A.M.F., Fontana C.A.P., Souza C.M. \& Stella A.E. 2010. Etiologia da mastite bovina subclínica, sensibilidade dos agentes às drogas antimicrobianas e detecção do gente da $\beta$-lactamase em Staphylococcus aureus. Vet. Zootec. 17:552-559.

Galdino M.C., Domingues P.F. \& Lapenna B.S.L. 2012. A produção de leite orgânico e aspectos de segurança alimentar. Vet. Zootec. 19:490-501.

Gopinath S.M., Suneetha T.B. \& Mruganka V.D. 2011. Chemical prophylaxis and antibacterial activity of methanolic and aqueous extracts of some medicinal plants against bovine mastitis. Int. J. Adv. Biol. Res. 1:93-95.

Haftu R., Taddele H., Gugsa G. \& Kalayou S. 2012. Prevalence, bacterial causes, and antimicrobial susceptibility profile of mastitis isolates from cows in large-scale dairy farms of Northern Ethiopia. Trop. Anim. Health Prod. 44:1765-1771.

Harmon R.J. \& Langlois B.E. 1989. Mastitis due to coagulase-negative Staphylococcus species. Agri-Practice 10.

Hayouni E.A., Miled K., Boubaker S., Bellasfar Z., Abedrabba M., Iwaski H., Iwaskie H., Okue H., Matsuie T., Limama F. \& Hamdi M. 2011. Hydroalcoholic extract based-ointment from Punica granatum L. peels with enhanced in vivo healing potential on dermal wounds. Phytomedicine 18:976-984.

Ismail T., Sestili P. \& Akhtar S. 2012. Pomegranate peel and fruit extracts: A review of potential anti-inflammatory and anti-infective effects. J. Ethnopharmacol. 143:397-405.

Jain P. \& Nafis G. 2011. Antifungal activity and phytochemical analysis of aqueous extracts of Ricinus communis and Punica granatum. J. Pharmacy Res. 4:128-129.

Langoni H. 2013. Qualidade do leite: utopia sem um programa sério de monitoramento da ocorrência de mastite bovina. Pesq. Vet. Bras. 33: 620-626.

Lansky E.P. \& Newman R.A. 2007. Punica granatum (pomegranate) and its potential for prevention and treatment of inflammation and cancer. J. Ethnopharmacol. 109:177-206.

Lee C.J., Chen L.G., Liang W.L. \& Wanga C.C. 2010. Anti-inflammatory effects of Punica granatum Linne in vitro and in vivo. Food Chemistry 118:315-322.

Machado T.B., Pinto A.V., Pinto M.C.F.R., Leal I.C.R., Silva M.G., Amaral A.C.F., Kuster R.M. \& Netto-dos-Santos K.R. 2003. In vitro activity of Brazilian medicinal plants, naturally occurring naphthoquinones and their analogues, against methicillin-resistant Staphylococcus aureus. Int. J. Antimicrob. Agents 21:279-284.

Manasathien J., Indrapichate K. \& Intarapichetet K. 2012. Antioxidant activity and bioefficacy of pomegranate Punica granatum Linn. peel and seed extracts. Glob. J. Pharmacology 2:131-141.

Manian R., Anusuya N., Siddhuraju P. \& Manian S. 2008. The antioxidant activity and free radical scavenging potential of two different solvent extracts of Camellia sinensis (L.) O. Kuntz, Ficus bengalensis L. and Ficus racemosa L. Food Chemistry 107:1000-1007.

Marques V.F., Souza M.M.S., Mendonça E.C.L., Alencar T.A., Pribul B.R., Coelho S.M.O., Lasagno M. \& Reinoso E.B. 2013. Análise fenotípica e genotípica da virulência de Staphylococcus spp. e de sua dispersão clonal como contribuição ao estudo da mastite bovina. Pesq. Vet. Bras. 33:161-170. 
Mello-Peixoto E.C.T., Jardim J.G., Heinzen E.L., Domingues P.F., Padovani C.R. \& Oliveira Orsi R. 2012. Própolis no controle da mastite bovina. Archs Vet. Sci. 17:43-52.

Menezes S.M.S., Pinto D.M. \& Cordeiro L.N. 2008. Atividades biológicas in vitro e in vivo de Punica granatum L. (romã). Revta Bras. Med. 65:388391.

Moorthy K., Punitha T., Vinodhini R., Thippan B., Sureshkumar B.T. \& Vijayalakshmi P. 2013. Antimicrobial activity and qualitative phytochemical analysis of Punica granatum Linn. (PERICARP). J. Med. Plants Res. 7:474-479.

Morais S.M., Lima K.S.B., Siqueira S.M.C., Cavalcanti E.S.B., Souza M.S.T., Menezes J.E.S.A. \& Trevisan M.T.S. 2013. Correlação entre as atividades antiradical, antiacetilcolinesterase e teor de fenóis totais de extratos de plantas medicinais de farmácias vivas. Revta Bras. Plantas Medicinais 15:575-582.

Nickerson S.C. 1993. Preventing new Staphylococcus aureus infections. Vet. Med. 88:368-374.

Noda Y., Kaneyuki T., Mori A. \& Packer L. 2002. Antioxidant activities of pomegranate fruit extract and its anthocyanidins: delphinidin, cyanidin, and pelargonidin. J. Agricult. Food Chem. 50:166-171.

Oliveira L.P., Pinheiro R.C., Vieira M.S., Paula J.R., Bara M.T.F. \& Valadares M.C. 2010. Atividade citotóxica e antiangiogênica de Punica granatum L., Punicaceae. Revta Bras. Farmacogn. 20:201-207.

Oliveira C.M.C., Souza M.G.S., Silva N.S., Mendonça C.L., Silveira J.A.S., Oaigen R.P., Andrade S.J.T. \& Barbosa J.D. 2011. Prevalência e etiologia da mastite bovina na bacia leiteira de Rondon do Pará, estado do Pará. Pesq. Vet. Bras. 31:104-110.

Ondiek J.O., Ogore P.B., Shakala E.K. \& Kaburu G.M. 2013. Prevalence of bovine mastitis, its therapeutics and control in Tatton Agriculture Park, Egerton University, Njoro District of Kenya. Basic Res. J. Agricult. Sci. Rev. 2:15-20.

Pedrini S.C.B. \& Margatho L.F.F. 2003. Sensibilidade de microrganismos patogênicos isolados de casos de mastite clínica em bovinos frente a diferentes tipos de desinfetantes. Arqs Inst. Biológico, São Paulo, 70:391-395.

Pereira J.V., Pereira M.S.V., Sampaio F.C., Sampaio M.C.C., Alves P.M., Araújo C.R.F. \& Higino J.S. 2006. Efeito antibacteriano e antiaderente in vitro do extrato da Punica granatum Linn. sobre microrganismos do biofilme dental. Revta Bras. Farmacogn. 16:88-93.

Pereira A.V., Rodrigues O.G., Azevêdo T.K.B., Bezerra D.A.C., De Lima E.Q. \& Pereira M.S.V. 2009. Perfil de extrato de plantas sobre Staphylococcus aureus isolado de mastite bovina. Revta Biol. Farmácia 3:1-5.

Pinto M.S., Faria J.E., Message D., Cassini S.T.A., Pereira C.S. \& Gioso M.M. 2001. Efeito de extratos de própolis verde sobre bactérias patogênicas isoladas do leite de vacas com mastite. Braz. J. Vet. Res. Anim. Sci. 38:278-283.

Rajan S., Ravi J., Suresh A. \& Guru S. 2013. Hidden secrets of Punica granatum use and its effects on oral health: a short review. J. Orofacial Res. 3:38-41.

Raza A., Muhammad G., Sharif S. \& Atta A. 2013. Biofilm producing Staph- ylococcus aureus and bovine mastitis: a review. Molec. Microbiol. Res. 3:1-8.

Ribeiro M.G., Geraldo J.S., Langoni H., Lara G.H.B., Siqueira A.K., Salerno T. \& Fernandes M.C. 2009. Microrganismos patogênicos, celularidade e resíduos de antimicrobianos no leite bovino produzido no sistema orgânico. Pesq. Vet. Bras. 29:52-58.

Rummun N., Somanah J., Ramsaha S., Bahorun T. \& Neergheen-Bhujun V.S. 2013. Bioactivity of nonedible parts of Punica granatum L.: a potential source of functional ingredients. Int. J. Food Sci. 2013:1-12.

Ruegg P.L. 2003. Practical food safety interventions for dairy production. J. Dairy Sci. 86(Suppl.):E1-E9.

Sabour P.M., Gill J.J., Lepp D., Pacan J.C., Ahmed R., Dingwell R. \& Leslie K. 2004. Molecular typing and distribution of Staphylococcus aureus isolates in Eastern Canadian dairy herds. J. Clin. Microbiol. 42 :3449-3455.

Saeki E.K., Mello-Peixoto E.C.T., Matsumoto L.S., Marcusso P.F. \& Monteiro R.M. 2012. Mastite bovina por Staphylococcus aureus: sensibilidade às drogas antimicrobianas e ao extrato alcoólico de própolis. Acta Vet. Bras. 5:284-290.

Santos L.L., Pedroso T.F.F. \& Guirro E. 2010. Perfil etiológico da mastite bovina na bacia leiteira de Santa Izabel do Oeste, Paraná. Ciênc. Anim. Bras. 11:860-866

Saraiva R.M.C. 2012. Atividade antibacteriana de plantas medicinais frente a bactérias multirresistentes e a sua interação com drogas antimicrobianas Dissertação de Mestrado em Ciências Farmacêuticas, Instituto de Ciências da Saúde, Universidade Federal do Pará, Belém, PA. 67p.

Schleifer K.H. \& Kloos W.E. 1975. A simple test system for the separation of staphylococci from micrococci. J. Clin. Microbiol. 1:337-338.

Silva M.A.R., Higino J.S., Pereira J.V., Siqueira-Júnior J.P. \& Pereira M.S.V. 2008. Antibiotic activity of the extract of Punica granatum Linn. over bovine strains of Staphylococcus aureus. Revta Bras. Farmacogn. 18:209-212.

Silva B.T., Anjos C., Novo S.M.F., Matsumoto L.S., Mello-Peixoto E.C.T., Silva L.P. \& Silva R.M.G. 2013. Atividade antimicrobiana in vitro de extrato de Punica granatum L. sobre Staphylococcus aureus isolado em leite bovino. Biosci. J. 29:974-984.

Souza C.M.P., Brandão D.O., Silva M.S.P., Palmeira A.C., Simões M.O.S. \& Medeiros A.C.D. 2013. Utilização de plantas medicinais com atividade antimicrobiana por usuários do Serviço Público de Saúde em Campina Grande-Paraíba. Revta Bras. Plantas Medicinais 15:188-193.

Tozzetti D.S., Bataier M.B.N., Almeida L.R. \& Piccinin A. 2008. Prevenção, controle e tratamento das mastites bovinas: revisão de literatura. Revta Cient. Eletr. Med. Vet. 6:10.

Trevisan L.F.A., Pereira A.V., Albuquerque A.C.L., Pereira M.S.V., Pereira J.V., Rodrigues O.G., Lima E.Q. \& Melo M.A. 2009. Resistência microbiana aos fármacos no tratamento de mastites: alternativas naturais para romper essa barreira. Revta Agropec. Téc. 30:51-56.

Werkman C. \& Granato D. 2008. Aplicações terapêuticas de Punica granatum L. (romã). Revta Bras. Plantas Medicinais 10:104-111.

Zhishen J., Mengcheng T. \& Jianming W. 1999. The determination of flavonoid contents in mulberry and their scavenging effects on superoxide radicals. Food Chemistry 64:555-559. 